\title{
INTEGRATION OF SENTINEL-2 AND LANDSAT-8 DATA FOR SURFACE REFLECTANCE TIME-SERIES ANALYSIS
}

\author{
G. Berdou, S. Shrestha, M. Hahn
}

Dept. of Geomatics, Computer Science and Mathematics Stuttgart University of Applied Sciences Stuttgart, Germany - gabrielberdou@gmail.com; shrestha.suress@gmail.com; michael.hahn@hft-stuttgart.de

KEY WORDS: Atmospheric correction, Landsat 8 \& Sentinel-2 data integration, surface reflectance, remote sensing, quality assessment.

\begin{abstract}
:
Integration of Sentinel-2 and Landsat-8 imagery is a key factor to provide earth observation data at a global scale with higher temporal resolution. Integration of data from two sensors is possible with the consistent harmonized data framed in common reference and processing, which can be used for comparing geophysical surface characteristics. This study focuses on the analysis of the atmospheric correction methods available for both Landsat-8 and Sentinel-2 products to convert the top of the atmosphere to the bottom of atmosphere reflectance. Other investigations (De Keukelaere, 2018) carried out similar analyses focusing on data acquired over water, while this study emphasises the analyses over land covers. Two processing algorithms iCOR and Sen2COR are utilized to perform atmospheric corrections, and results are statistically and visually compared. Comparisons based on same images processed with different algorithms show very strong correlation for some classes (urban: 0.99), while correlation values around 0.85 were achieved between images from different sensors.
\end{abstract}

\section{INTRODUCTION}

Sentinel-2 and Landsat 8 missions provide earth observation data at a global scale, with comparable centre wavelength and bandwidths. Level 2 data for both satellites are atmospherically corrected surface reflectance products, processed using different algorithms.

Harmonized L8 and S2 surface reflectance products increase consistently the temporal scope for time series analysis since Landsat missions offer coverage for almost five decades, and Sentinel 2 missions (A and B) increases the temporal resolution up to 5 days. The combination of both data products can be gridded to a common reference frame and processed to compare geophysical parameters, regardless of the sensor of origin. Harmonization is achieved by the utilization of algorithms to obtain seamless products like atmospheric corrections, cloud and cloud shadow masking, spatial co-registration and common gridding, bidirectional reflectance distribution and bandpass adjustment (Claverie, 2012).

The main objective of this paper is to study the integration of S2 and L8 data for time series surface reflectance analysis. The study focuses on the atmospheric correction methods available for both Landsat- 8 and Sentinel-2 products.

The atmosphere is a major agent of distortion for spaceborne images, provoking scattering and absorption, the molecules and aerosols in the atmosphere alter the reflectance from the earth's surface. In this context, the atmospheric correction has significant influence on the process of obtaining reflectance values from satellite images.

\subsection{Theoretical background}

The atmospheric correction algorithm calculates Bottom of Atmosphere (BOA) reflectance from Top of Atmosphere (TOA) reflectance based on a combination of atmospheric, topographic and reflectance data. Sentinel level 2A (atmospherically corrected) data is based on a methodology proposed in Atmospheric Correction for Satellite Imagery (Richter, 2011), which performs corrections utilizing the LIBRADTRAN radiative transfer model.

The LIBRADTRAN radiative transfer model provides a large database of Look Up Table (LUT) that covers a wide range of atmospheric conditions, solar geometries and ground elevations. The tables are generated with high spectral resolution $(0.6 \mathrm{~nm})$ and then resampled with SENTINEL-2 resolutions. They serve as a simplified model to invert the radiative transfer equation to calculate BOA reflectance (Mayer, 2005). All gaseous and aerosol properties of the atmosphere are either derived by the algorithm itself or fixed to an a priori value.

The Aerosol Optical Thickness (AOT) can be derived from the images themselves above reference areas of known reflectance behaviour, preferably Dark Dense Vegetation (DDV) targets, and water bodies.

If no sufficiently dark reference areas can be found in the scene, an incremental threshold in the $2.2 \mu \mathrm{m}$ band is applied (from 0.05 to 0.1 and 0.12 ) to identify reference areas with medium brightness.

The visibility, and consequently the corresponding AOT, is automatically derived from a correlation of SWIR reflectance (band $12=2.19 \mu \mathrm{m}$ ) with the blue (band 2) and red (band 4) reflectance. The principle of the AOT retrieval method is based on Kaufman et al. (1997) with some slight differences (reduction of negative reflectance values, fixed rural/continental aerosol type, etc.). Sentinel-2 atmospheric correction employs Lambert's reflectance law. Topographic effects can be corrected during the surface retrieval process using an accurate Digital Elevation Model (DEM).

Two processing algorithms for atmospheric correction, namely Sen2Cor processor from Telespazio France and iCOR from 
VITO Belgium, available in SNAP software, are evaluated in this paper for feasibility of S2 and L8 data integration.

\section{OBJECTIVES}

The main objectives of the study are the

- Assessment of the integration of Sentinel-2 and Landsat-8 imagery for increasing time-related accuracy.

- Evaluation of the differences between two processors to perform atmospheric correction, iCOR and Sen2cor, particularly with readily available Sentinel-2 images.

- Comparison of the difference in reflectance values from the two processors, both temporally and spatially, for an urban area in Stuttgart, Germany.

\section{METHODOLOGY}

\subsection{Study area and dates}

An area of interest of $580 \mathrm{~km}^{2}$ containing the city of Stuttgart was defined, using WGS_1984_UTM_Zone_32N as coordinate reference system.

The time series aims to cover the four seasons of the year, in order to assess the influence of different temperature and weather conditions over reflectance values.

\begin{tabular}{|c|c|}
\hline Sentinel-2 & Landsat 8 \\
\hline $2018 / 05 / 07$ & $2018 / 05 / 18$ \\
\hline $2018 / 08 / 20$ & $2018 / 08 / 22$ \\
\hline $2018 / 11 / 18$ & - \\
\hline $2019 / 02 / 24$ & $2019 / 02 / 23$ \\
\hline
\end{tabular}

Table 1. Image sensing dates.

For the Landsat 8 images in autumn, no free cloud coverage images were found, so the analysis and comparisons concerning Landsat 8 are performed with three images only, while those for Sentinel-2 involves four images from different dates.

\subsection{Methodology overview}

The atmosphere modifies the radiation measured by optical sensors. By the means of atmospheric correction, the Top of Atmosphere (TOA) reflectance values can be transformed into Bottom of atmosphere values (BOA) by considering the influence of Aerosol Optical Thickness (AOT), water content of the atmosphere and terrain characteristics.

The overall methodology consists of the acquisition of both processed and unprocessed images, the definition of a temporal and spatial work frame (dates and study area), processing of the satellite images which includes: atmospheric corrections with two different processors, cloud masking, supervised classification for land cover classification, band extraction and data clipping to study area subset. Finally, a comparison of reflectance values is performed by statistical means such as correlation and covariance matrices, variation coefficients and mean values, applied with an algorithm developed in python.

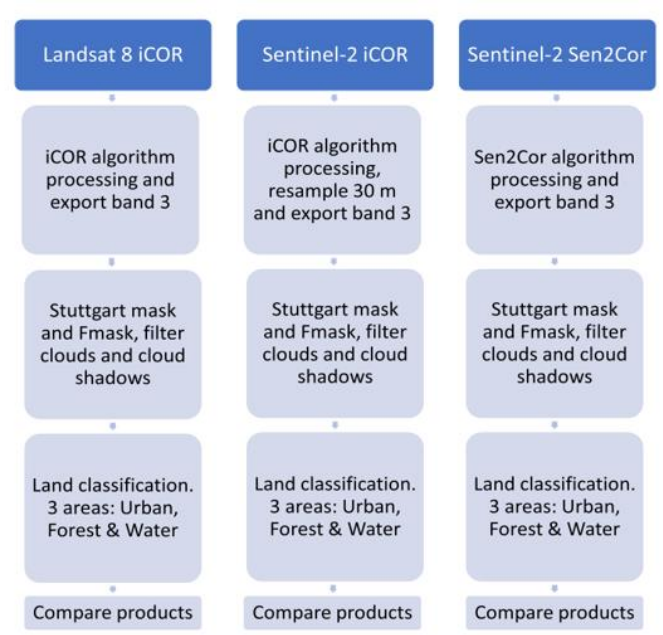

Figure 1. Diagram of data processing workflow

\subsection{Cloud masking}

Sentinel-2 level 2 and LC1 images are first treated with a cloud masking algorithm. Pixels belonging to cloud and cloud shadow are filtered out, and those corresponding to clear land are kept for processing.

Fmask algorithm is based on a set of TOA reflectance thresholds to classify clouds, cloud shadows, water, and snow, it relies on cloud and cloud shadow matching using sun-view geometry information. Since it was initially designed for Landsat data, the algorithm relies on thermal data. An adapted version of Fmask to work in different configurations with options to omit thermal and/or cirrus bands is also available and is used to treat Sentinel-2 data. The per-pixel sun-view geometry information is derived from the L1C metadata (Claverie, 2012).

\subsubsection{Sen2Cor processor overview}

Sen2Cor processor was developed by Telespazio France, with strong support of DLR (German Spatial Agency) for the validation, the organization of in-situ campaigns and the collection of reference data for validation.

Sentinel-2 level 2 processing is applied to granules of TOA Level-1C ortho-image reflectance products. The processing starts with the Cloud Detection and Scene Classification followed by the retrieval of the AOT and the Water Vapour (WV) content from the L1C image. The final step is the TOA to BOA conversion.

Sen2Cor also includes several optional processing parameters that can be utilized such as cirrus correction, terrain correction, adjacency correction, and empirical BRDF-corrections. Sen2Cor relies on two main auxiliary data: Radiative Transfer Look-Up Tables and the Digital Elevation Model (DEM). Sen2Cor processor is only available to process Sentinel-2 images and can be accessed as a plugin installed in SNAP image processing software. 


\section{4 iCOR processor overview}

The iCOR atmospheric correction processor is developed and maintained by the Remote Sensing group, from the Vlaams Instituut voor Technologisch Onderzoek (VITO).

iCOR stands for image correction for atmospheric effects and it was designed to work over inland, coastal or transitional waters and land, it depends on auxiliary data for the atmospheric correction, which can be originated from external sources or be derived from the image itself. External auxiliary data such as digital elevation model (DEM), solar and viewing angles and the atmospheric composition are utilized in the process of correction.

The satellite overpass time, sensor and sun position provides information on solar and viewing angles. The atmospheric composition model iCOR processor relies on, is described by a combination of an aerosol model and the aerosol optical thickness (AOT), which indicates how much direct sunlight is prevented from reaching the ground by these aerosol particles (De Keukelaere, 2018).

This algorithm corrects adjacency effects, which consists of light originating from neighboring pixels and scattered into the target-sensor path (reflection from contiguous pixels).

Atmospheric components, once assessed, are incorporated into a Radiative Transfer Model. In the iCOR processor, the model uses Moderate-Resolution Atmospheric Radiance and Transmittance Model (MODTRAN5), which assumes that the radiance received by the sensor consits of the atmospheric path radiance, the background path radiance (adjacency effects) and the radiance from the target (De Keukelaere, 2018).

Atmospheric corrections can be performed on both Sentinel-2 and Landsat 8 level 1 images. This allows comparative analyses between reflectance values for both sensors and thus improves the temporal resolution for time series analysis. Since the spatial resolution is different for both sensors, it is necessary to resample Sentinel-2 images of $10 \mathrm{~m}$ resolution to $30 \mathrm{~m}$ in order to produce homologous products from both sensors.

\section{RESULTS}

The output of the atmospheric correction for the implemented processors is BOA reflectance values derived from TOA values. Different processors, by changing methodology and treatment algorithms of the raw input images, are expected to produce different results, which are further visualized and analyzed for assessment and quantification, by means of statistical methods.

In order to evaluate the influence of different land cover on the output reflection values, three representative areas for the classes forest, open land and urban area are defined.

Due to the number of images (four images S2A, four images LC1 and three images L8 Level 1) and the different processor outputs (namely four Sen2Cor outputs and seven iCOR outputs), the analysis is confined to selected dates and areas of interest. The Sentinel-2 images are processed using Sen2Cor and iCOR algorithms, while Landsat 8 images are only processed with iCOR.

\subsection{Sentinel-2: iCOR and Sen2Cor processors comparison}

First, differences between Sentinel-2 images processed with Sen2Cor and iCOR are assessed by subtracting the reflectance values from one processor to the other to visualize the difference between both outputs.

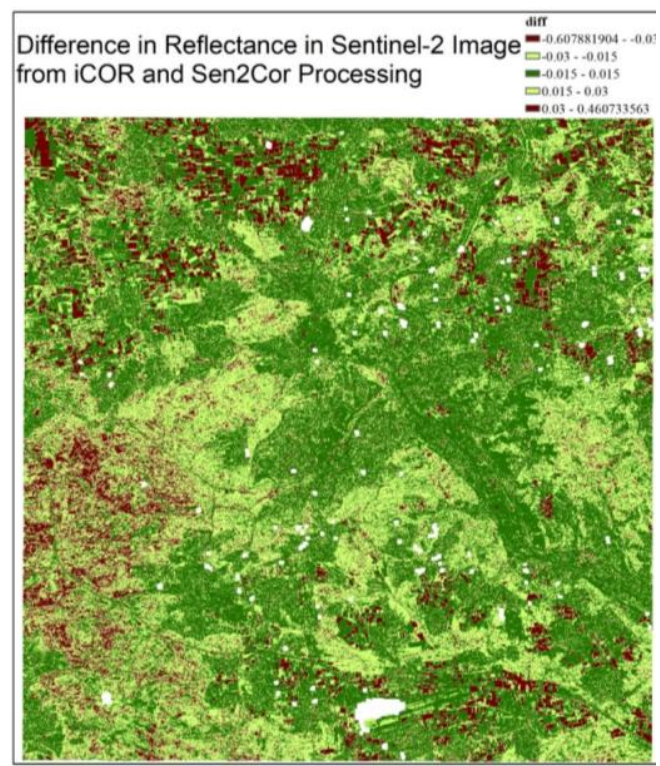

Figure 2. Sentinel-2: iCOR and Sen2Cor reflectance value differences.

Highest differences between reflectance values (both positive or negative) are visualized with red colour, differences in the range of 0.015 to 0.03 are symbolized with light green, while differences up to 0.015 with dark green.

The histogram (Figure 3 ) shows that the differences between the reflection values for both processors are relatively small. The pixel by pixel difference comparison reveals that most differences lie between -0.035 to 0.035 . The largest differences occur in the open/cultivated areas.

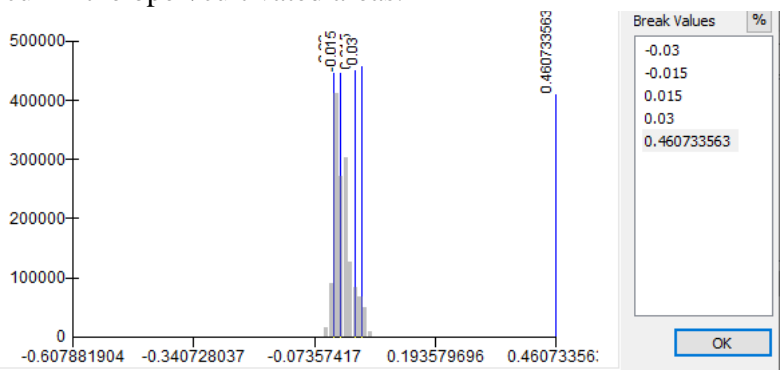

Figure 3. Histogram, iCOR \& Sen2Cor reflectance differences.

To evaluate the correlation between the outputs of both processors, the values are analysed with a scatterplot by displaying Sen2Cor values in the Y-axis and iCOR values in the $\mathrm{X}$-axis. The standard deviations of the Sen2Cor processor are between 0.03-0.04 and for iCOR processor between 0.04-0.05.

The correlation between reflectance values of two processors is $95.1 \%$. 


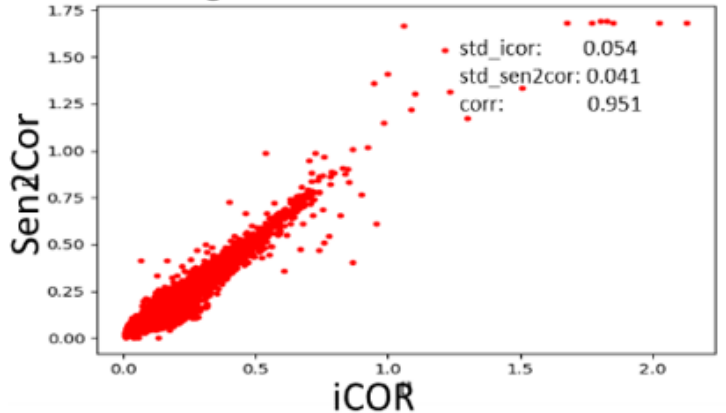

Figure 4. Scatterplot, iCOR \& Sen2Cor difference reflectance. Stuttgart area 2018/05

The scatterplot shows some artifacts when the reflectance values are higher than 0.5 , and visual analysis shows that these artifacts are distributed over urban areas, in very small numbers i.e. just single pixels distributed over an area. Red points in the image below show pixels with a reflectance difference bigger than 0.5 for both processors. A detailed inspection with an RGB natural color combination of Sentinel-2 images indicates that those reflections are from metal surfaces (such as aluminium roofs or solar panels).

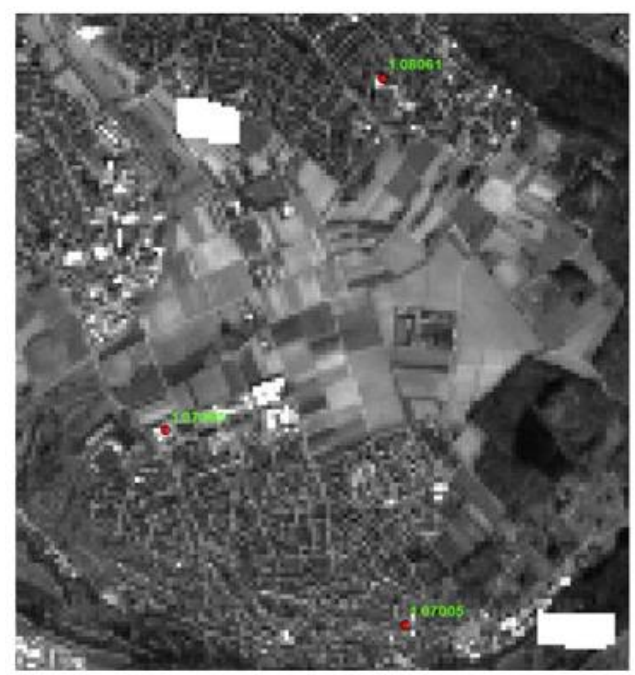

Figure 5. Location of artefacts found between iCOR and Sen2Cor reflectance values.

Two areas, representing two main land cover classes of the scene (urban and forest), are defined utilizing the S2 RGB image. In order to assess the reflectance differences of the iCOR and Sen2Cor results, the reflectance values for each class are compared with a scatter diagram, which shows an almost perfect correlation (0.99) in urban areas and a strong correlation in forest areas (0.86). Urban areas show much higher reflectance values $(>0.125)$ than forest areas $(<0.05)$, due to the spectral response of urban sealed surfaces. Correlation between iCOR and Sen2Cor is stronger in urban than in forest areas. A concomitant increase pattern is observed between reflectance values and correlation between the analysed atmospheric correction processors: in those areas where reflectance values are higher than 0.15 (predominantly urban areas), correlation coefficient between processors tends to 1 .

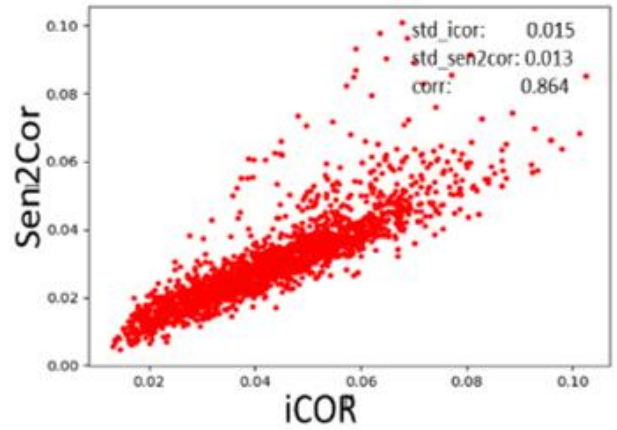

Figure 6. Scatterplot for Sen2Cor and iCOR in forest area. 2018/05

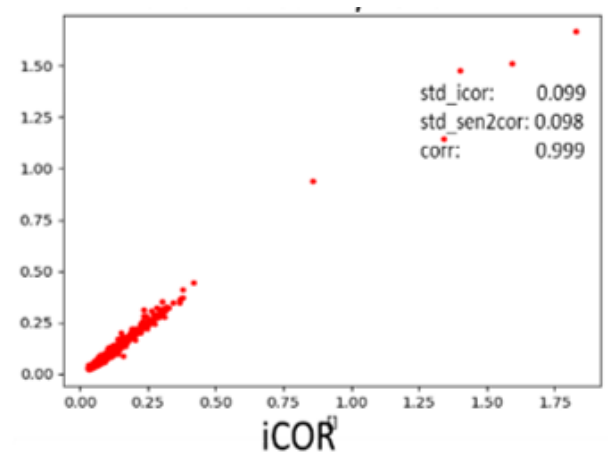

Figure 7. Scatterplot for Sen2Cor and iCOR in urban area. 2018/11.

Variation in reflectance values is analysed for single pixels, between both processor and S2 Level 2A data (in the histogram below presented in blue: class 'sen'). Pixels corresponded to 3 classes: urban, forest and water.

The visualization of the differences with a histogram shows that pixels representing water and forest have on average low reflectance values ( $<0.05$ for forest, and $<0.025$ for water). This can be are explained by the low spectral response of these elements in band 3 (central wavelength $559.8 \mathrm{~nm}$ ). The classes present a maximum variation between them of 0.025 in reflectance value.

For the pixel representing the urban class, there is a pronounced difference of 0.075 in the reflectance value between iCOR and Sen2Cor.

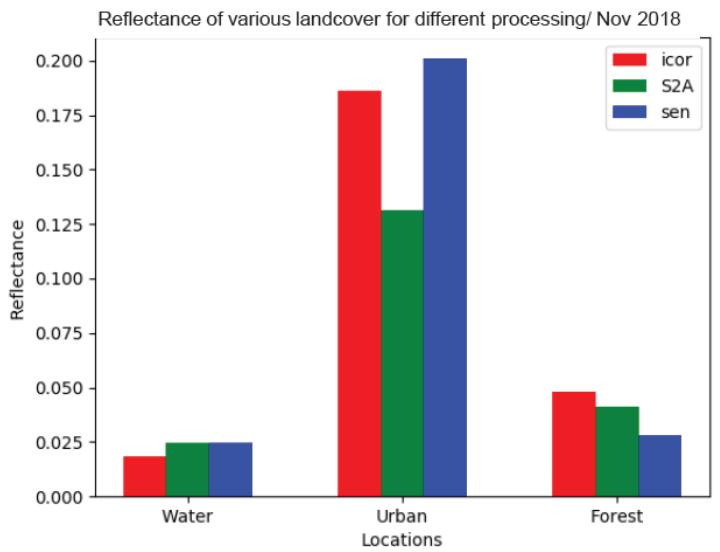

Figure 8. Histogram of pixel reflectance values for land use. 


\subsection{Sentinel-2 \& Landsat 8: iCOR processor comparison}

The atmospheric correction of both Landsat 8 and Sentinel-2 raw images can be made with the iCOR processor, applying similar, but no identical procedures (since Sentinel-2 does not have thermal bands; the cirrus band is used instead in the process of cloud and haze detection). Raw images are atmospherically corrected and compared.

The differences between reflectance values from L8 and S2 processed with iCOR are analysed to highlight those areas which show the greatest difference in reflectance values.

Before the differences are calculated, the $\mathrm{S} 2$ data are resampled using a bilinear interpolation that changes the spatial resolution of band 3 to $30 \mathrm{~m}$ (to adjust the spatial resolution of band 3 in L8). To exclude the pixels containing clouds and haze from the analysis, a cloud mask is created which adds the mask of L8 to that of S2 to process only the pixels belonging to clear land in both images.

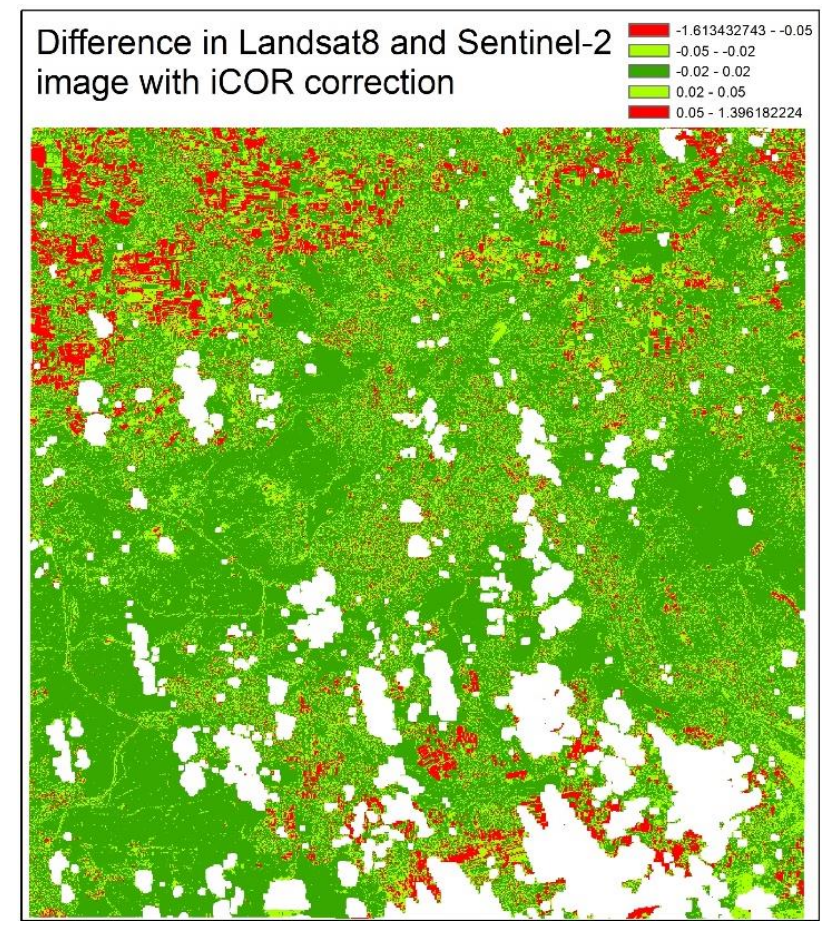

Figure 9. Landsat 8 \& Sentinel-2 reflectance values difference with iCOR.

In Figure 9, the red pixels represent the areas with larger differences $(>0.05)$ between the two images. These are in the north and southeast of the study area. Dark green shows the lowest differences between both images (less than 0.02), while light green depicts those areas with an intermediate level of difference (between 0.02 to 0.05 ).
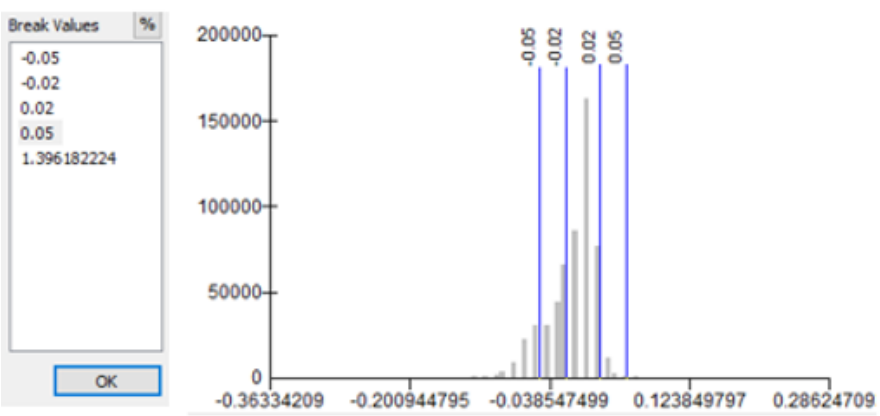

Figure 10. Histograms with values from Landsat 8 and Sentinel2 corrected with iCOR.

The histogram of Figure 10 depicts the distribution of differences between both images, showing that most pixel values (differences in reflection values) oscillate between -0.05 and 0.05 .

A supervised image classification is performed in the study area, and sample areas are defined for the classes: river, lake, forest, agricultural fields, open land and urban infrastructure.

The comparison between classified image and difference image shows that the open land and agricultural fields correspond to those areas in which the reflectance differences of both images are higher than 0.05 .

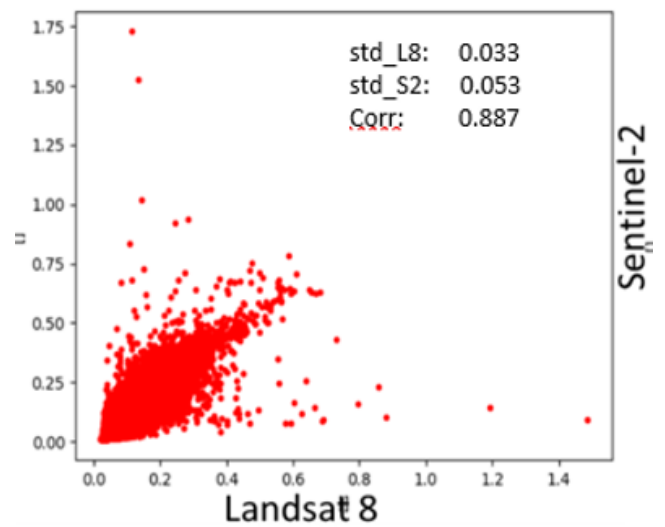

Figure 11. Scatterplot Landsat 8 and Sentinel-2 corrected with iCOR, images of both sensors for 2018/08.

The scatterplot from the figure above shows a high correlation between both processed images. The values where correlations are weaker but above the threshold of 0.4 correspond to highly reflective urban areas associated with roof tops of different materials. 


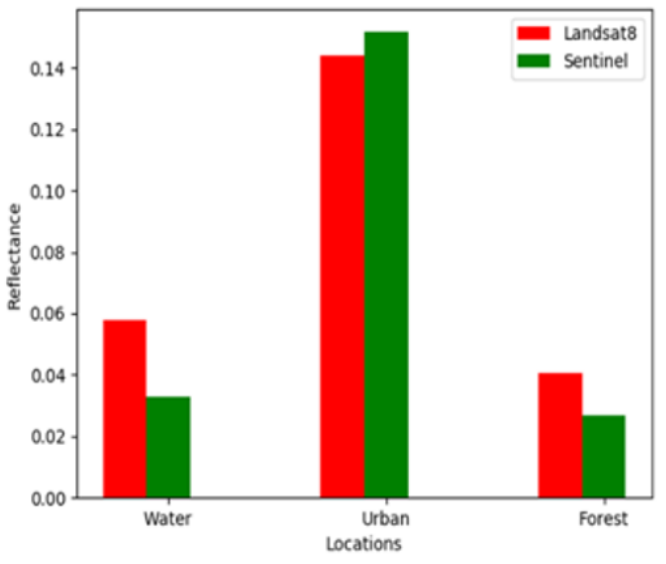

Figure 12. Landsat 8 and Sentinel-2 data corrected with iCOR values for Land Cover.

Figure 12 shows a comparison of the reflectance values generated with iCOR for L8 and for S2. Due to the use of spectral band 3, higher reflectance values correspond to urban areas. As in the comparison between iCOR and Sen2Cor, the difference between L8 and S2 is smaller in urban areas, while in those areas where reflectance values are lower (water and forest) the difference between images increases.

\section{CONCLUSIONS}

The purpose of this study was to assess the differences in reflectance values generated by two atmospheric correction methods and from L8 and S2 imagery for different land covers. In analyzing the reflectance of images, the study has encountered some limitations. Due to the high cloud coverage presented from autumn to spring in the study area (availability of cloud free images was near zero), a minimum of $10 \%$ cloud coverage was used when searching for images in the web repositories. Landsat- 8 and Sentinel- 2 image could not be found on the same date, so when analyzing the atmospheric corrections between L8 and S2 no identical atmospheric conditions prevail, which at least slightly affect the investigations.

When comparing processors working on the same input images (Sentinel-2), it was found that a strong correlation exists (greater than 0.85) for the two main land covers urban and forest, and that correlation values increase in areas where reflectance values are higher, although some extreme differences appear in those areas with very high reflectance values or abnormal spectral behaviour.

The analysis of L8 and S2 images that were atmospherically corrected with the same processor (iCOR) showed an overall strong correlation (0.88). With the correlation analysis between the land classification classes, the higher differences in the reflectance values $(>0.05)$ were found in the classes open area and agricultural fields.

These results are promising for the integration of surface reflectance values, both from the two processors iCOR and Sen2Cor as well as from L8 and S2 products. For S2 and L8 products obtained with iCOR, certain caution is necessary when comparing classes such as agricultural fields and open land areas, as a difference of $5 \%$ or even higher is expected.
Further analysis of reflectance values for L8 and S2 products based on other spectral bands and land cover may follow this work. It can be expected that the accuracy of the multi-temporal analysis that integrates the data from both sensors, will continue to improve.

\section{ACKNOWLEDGEMENT}

This publication was produced in the project „Neue Möglichkeiten der Datenerhebung, -analyse und Auswirkungsabschätzung im Wasser-Energie Nexus - NeMDa“, which is part of the Baden-Württemberg-STIPENDIUM for University Students - BWS plus, a programme of the Baden-Württemberg Stiftung. The authors would like to thank the BadenWürttemberg Stiftung for its support.

\section{REFERENCES}

Claverie, M., ju, j., Masek, J., Dungan, J., Vermote, E., Roger, J.-C., Skakun, S. and Justice, C., 2018. The Harmonized Landsat and Sentinel-2 surface reflectance data set. Remote Sensing of Environment, Vol. 219, pp. 145-161.

Keukelaere, L. de, Sterckx, S., Adriaensen, S., Knaeps, E., Reusen, I., Giardino, C., Bresciani, M., Hunter, P., Neil, C., van der Zande, D. and Vaiciute, D., 2018. Atmospheric correction of Landsat- 8/OLI and Sentinel-2/MSI data using iCOR algorithm: validation for coastal and inland waters. European Journal of Remote Sensing, Vol. 51 No. 1, pp. 525-542.

Louis, J., et al., 2016. Sentinel-2 SEN2COR: L2A processor for users. Proceedings of the Living Planet, Prague, Czech Republic.

Mayer, B. and Kylling, A., 2005. Technical note: The libRadtran software package for radiative transfer calculations description and examples of use. Atmos. Chem. Phys., 5, 18551877, https://doi.org/10.5194/acp-5-1855-2005.

R. Richter and Schläpfer, D., 2011. Atmospheric/Topographic Correction for Satellite Imagery: ATCOR-2/3 UserGuide, DLR IB 565-01/11, Wessling, Germany.

Sentinel-2 L2A data, 2019. Atmospheric Correction procedures. https://earth.esa.int/web/sentinel/technical-guides/sentinel-2msi/level-2a/algorithm (30.07.2019)

Smith, J., 2000. Remote sensing to predict volcano outbursts. Int. Arch. Photogramm. Remote Sens. Spatial Inf. Sci., XXVIIB1, 456-469. 